18 | 2014

NOVECENTO... E DINTORNI

Da Torino a Parigi: Laura Malvano storica e critica d'arte

\title{
La passione di Laura per l'arte: le radici familiari
}

La passion de Laura pour l'art : les racines familiales

How Laura Developed a Passion for the History of Art: The Influence of Her

Parents

Pino Mantovani

\section{(2) OpenEdition}

\section{Journals}

Edizione digitale

URL: http://journals.openedition.org/cei/1550

DOI: 10.4000/cei.1550

ISSN: 2260-779X

Editore

UGA Éditions/Université Grenoble Alpes

\section{Edizione cartacea}

Data di pubblicazione: 30 mars 2014

Paginazione: 63-71

ISBN: 978-2-84310-268-4

ISSN: 1770-9571

Notizia bibliografica digitale

Pino Mantovani, «La passione di Laura per l'arte: le radici familiari», Cahiers d'études italiennes [Online], 18 | 2014, online dal 30 septembre 2015, consultato il 28 mars 2021. URL: http://

journals.openedition.org/cei/1550 ; DOI: https://doi.org/10.4000/cei.1550 


\title{
LA PASSIONE DI LAURA PER L'ARTE: \\ LE RADICI FAMILIARI
}

\author{
Pino Mantovani
}

Ugo Malvano e Nella Marchesini si sposarono nel settembre del I930: fu l'incontro di due "anime sorelle» ${ }^{\mathrm{I}} \mathrm{ma}$ anche di storie molto diverse.

Nella, di famiglia toscana trasferita a Torino nel I9II: il padre, patriarca di biblica presenza, astratto nelle sue elucubrazioni, la madre, «arca del patto che custodisce le leggi», e «la tribù dei figlioli»; un'infanzia «selvaggia» a Marina di Massa, «estranea alle consuetudini», un'adolescenza silenziosa ed appartata, «ero suora», una giovinezza appassionata, «disarmonica»; condivide le emozioni con le sorelle differenti e complementari, ma l'intenzione di dare forma compiuta a sentimenti memorie desideri le fa seguire puntualmente quasi voracemente l'esempio del maestro Felice Casorati nel momento più alto del suo impegno artistico. La fine della giovinezza è sancita dalla morte del padre (I930) e poi della sorella Maria (1933). Il matrimonio segna l'avvio di un'altra epoca, caratterizzata anch'essa dalla presenza di una figura maschile di riferimento: ancora un padre, semmai meno esigente, non interessato ad imporsi come modello, anzi aperto al dialogo, a sua volta bisognoso di una controparte.

Ugo, di famiglia borghese, ricca, cittadina: un'adolescenza e una giovinezza non condizionate da rigide scadenze, forse con l'alibi di una certa precarietà di salute e di carattere (volitivo e dubbioso ad un tempo); studi impegnativi (di medicina) ma non così impegnati e convinti; autodidatta per vocazione e curiosità; artista per sensibilità prima che per abilità; viaggi (dopo la laurea, documentato fotograficamente quello in Egitto, concluso nel febbraio 1905), sport (scherma e montagna), quasi a rimandare un indirizzo e una scelta definitiva. Che cosa lega la montagna e la

I. Tutte le citazioni per le quali non è data altra indicazione sono tratte dallo "zibaldone» di Nella Marchesini, trascritto dalla nipote Maria Vittoria Malvano. 
pittura? Probabilmente l'emozione, la difficoltà della sfida, la disciplina e il metodo, alla fine un'immagine, guadagnata con fatica volontà destrezza. Parigi: emancipazione attraverso la scoperta di un mondo materiale e intellettuale variegato, fervido, affascinante; nella pittura, prima la luce esplosiva degli impressionisti, raggiunta quasi senza soluzione di continuità attraverso l'esperienza di un divisionismo non troppo sistematicamente applicato, poi la scoperta di Cézanne, che la luce carica di una responsabilità strutturale, tanto rispetto alle cose singole quanto rispetto allo spazio, non vuoto contenitore ma cosa più complessa ed articolata, comprensiva. Amore del grigio, da Corot a Cézanne, la convinzione che non si è pittori se non si è dipinto un grigio.

\section{I due sposi erano di generazioni ben distinte}

Ugo, del I878, appartiene ancora al XIX secolo avendo in esso consumato alcune esperienze fondamentali, fino alla cosiddetta Grande Guerra che segna duramente la sua maturità (volontario, eroe, ferito, si dissocia da ogni forma di violenza); sullo sfondo c'è la convinzione che la presa d'atto della realtà è un dovere, una responsabilità che si acumina nella scienza e nell'arte, c'è presto un'ideologia umanitaria che lo raggiunge tanto dagli studi medici quanto dall'arte. Insomma, la «realtà» è per Ugo Malvano, come per tutti i più impegnati della sua generazione, un termine di confronto prioritario: oggetto d'indagine positiva e di compromessa verifica. Il versante formale della pittura non costituisce invece problema prioritario, a meno che per forma non si intenda "procedimento adatto alla... visione, al temperamento ${ }^{2}$; di qui una certa incoerenza stilistica, modi e strategie condizionati dal raggiungimento dell'obbiettivo, che è la definizione non di ricalco dell'oggetto visivamente conosciuto: questo sì coerentemente caratterizzato, identico. Ci si può chiedere se la svolta cézanniana, compiuta nei primi anni Venti, sia stata di specie formale, o ancora nella direzione di un approfondimento conoscitivo. Qui si può dire che, data la costante ricerca di un metodo, quello di Cézanne, rigoroso come quello dei divisionisti, si presenta però meno meccanico e ripetitivo, meno pericolosamente esposto alla maniera, invece sostenuto dalla intenzione di «mantenere viva e sincera la prima visione [...], e l'amore che ci ha fatto vedere così in quel momento [...]» (ibid.). Ma su questo torneremo in seguito.

2. Lettera a Cino Bozzetti, da Drusacco, I8 agosto I947. 
Nella, nata nel I90I, è della generazione di artisti che a Torino costituiscono il nucleo della fronda filo-francese (Menzio, Chessa, Levi, Paulucci) che propone un rilancio del Postimpressionismo, forse del Fauvismo, anche sulla scorta delle indagini storico/teoriche di Lionello Venturi. Come pittrice, appartiene in tutto e per tutto al '900, non soltanto per data di nascita e avvio dell'attività dopo il discrimine della prima guerra mondiale, ma come tipo di ricerca, che parte dalla svolta casoratiana, fondata sulla consapevolezza che a nuovo contenuto deve corrispondere un linguaggio adeguato, espressivamente efficace. Una svolta che presuppone una secca presa di distanza dalla «realtà» come la poteva intendere un pittore dell'Ottocento, e un trasferimento dal piano ottico a quello dei significati. È utilissima, su questo vettore, l'interpretazione che propone del «maestro» (anche suo maestro) il pittore-filosofo-critico Albino Galvano, il quale coglie nell'esperienza simbolista (non solo di Casorati) un necessario passaggio antirealista, che mette i più intelligenti e decisi nella necessità di compiere un ulteriore passo, etico anziché semplicemente formale, che recuperi la magrezza e la purezza «funzionale», smarrita tanto dal naturalismo descrittivo o allegorico quanto dall'astrazione decorativa.

Nella Marchesini, di questa magrezza e purezza avverte da subito, per natura e cultura, la necessità: per lei l'immagine dev'essere semplice e senza ornamento, primitiva nella nudità dell'apparire, una primitività raggiunta con lunga ricerca:

Nei miei quadri quel che ho trovato poco a poco — scoperto attraverso prove, deciso con dubbi poi scartati — se da un verso toglie il nitore armonioso della costruzione di getto, incorpora dall'altra un essenziale approfondimento tirato su a poco a poco con mezzi di fortuna che imprimono un loro speciale senso originale al lavoro. Né preconcetti né ricordi — ma "provando e riprovando" per scartare le scorie e lasciare il limpido [...]

consapevole del fatto che «se il messaggio vuole essere inteso linguaggio d'arte deve parlare», dall'altra che l'arte "quasi fanciulla capricciosa volubile fugge e inganna / Fedele amatore. / Il grande faticoso lavoro (credi di averci impegnato l'anima) non degna di un cenno [...] / All'improvviso sorride a caso / su un fuggitivo casuale operare».

In questo senso (il termine sia assunto nella sua polivalenza) pesa anche il suo essere toscana, il suo volerlo essere, come sembra sottolineare il maestro che, non a caso, la ritrae emblematicamente «toscana» nella "Cenni»",

3. Allusione ad uno dei quadri più noti di Felice Casorati, Silvana Cenni. Si tratta di un ritratto di grandi dimensioni di una giovane donna il cui modello era appunto Nella Marchesini. Eco forse anche di Cennino 
Silvana per giunta che vuol dire Selvatica, Primitiva, forse dannunzianamente o forse sulla linea che Venturi sta elaborando fino a "Il gusto dei primitivi», dove "primitivo" non è chi non sa ma chi crede, chi ha la grazia di credere.

Malvano, a differenza della stanziale Marchesini, frequenta la Francia e in particolare Parigi per lunghi periodi, a cominciare dai primi anni del '900, tanto da tenervi studio in epoche e luoghi differenti. Potrebbe pertanto aver visto Cézanne, addirittura dalla mostra del 1907, quella, per intenderci, che tanto pesò nella vicenda dei Cubisti e degli Espressionisti francesi e che rivelò Cézanne a Rilke; ma non si può onestamente dire che il giovane pittore torinese, ancora alla ricerca di un'identità, abbia dimostrato in data tanto precoce interesse per il maestro di Aix; semmai, il fascino della pittura «etica» di Cézanne l'avvertì nel I920, visitando l'ampia e scelta mostra della Biennale di Venezia, organizzata da Pica con l'appoggio di Ojetti che aveva attinto nelle collezioni fiorentine di Fabbri e Loeser; la stessa Biennale che rivelò Cézanne a Casorati come "pittore della rinuncia». Ma è soprattutto a seguito di reiterate occasioni parigine nell'arco degli anni Venti, che Malvano elegge Cézanne suo riferimento privilegiato, come dimostrano alcune nature morte (veri e propri omaggi) e numerose vedute francesi e torinesi. Gli esiti più originali e maturi del suo cézannismo, Ugo li raggiunge, però, nella penultima ed ultima stagione, che si identificano con la guerra e l'immediato dopoguerra, per i pochi anni che gli è dato di sopravvivere, fino al ' 52 . Periodi particolarmente drammatici: nel ' 38 , infatti, le Leggi razziali fasciste cominciano a pesare sulla famiglia che si vede costretta a rifugiarsi nella Valchiusella, dove trascorre lunghi periodi, avendo, soprattutto durante il conflitto, scarsi rapporti con la città e semmai mantenendo corrispondenza con alcuni intimi, in particolare Cino Bozzetti, anche lui definitivamente isolato a Borgoratto Alessandrino. Drammatica la situazione per le non facili condizioni di sopravvivenza e per il pericolo immanente di arresto e deportazione, ma anche di straordinaria intensità. Si realizza infatti proprio in quegli anni quella totale concentrazione sul lavoro che Malvano aveva cercato per la vita intera, sempre tuttavia distratto da una sia pur discreta tentazione mondana e dalla possibilità di scelte esistenziali alternative (per esempio, Torino o Parigi? Vita separata o mondana?). Adesso, invece, una necessità oggettiva s'impone: non c'è alternativa, come in un certo senso era avvenuto a Cézanne una volta presa la decisione di lasciare Parigi e di

Cennini (I370-I440), pittore toscano contemporaneo di Masaccio e autore del primo trattato di pittura in volgare. 
tornare a Aix. Nel paesaggio semi-montano di Drusacco, Malvano, senza alcun artificio volontaristico, riconosce i motivi di una pittura tutta sua. Motivi come ragioni e motifs come occasioni visive in una natura generosa e severa. Trova, non senza difficoltà ma con intima soddisfazione, i suoi boschi, gli alberi, i viottoli e le stradine, le svolte, le baite, le case nude e semplici; in assenza dell'uomo, salvo che nelle tracce che ha lasciato in un tempo lungo che non è quello vissuto individualmente, tanto meno psichicamente, invece il tempo stesso delle piante (singole e dei boschi) e delle pietre (eventualmente organizzate secondo intelligenti esigenze, ma non per questo modificate nella loro natura). Le variazioni sono soprattutto di stagione e quindi di consistenza, di peso, per così dire sostantivali; sembrerebbe riproporsi la condizione del pittore immerso nella natura, che Malvano aveva cercato fin da giovane, insieme con Follini, Ubertalli, forse Tavernier nelle campagne piemontesi, poi con Maggi sulle montagne aostane e cuneesi. Ma c'è una differenza fondamentale: adesso, finalmente, Ugo si è lasciato veramente alle spalle la condizione di Monsieur Patissot, non è più il cittadino curioso di campagna e amante di montagna, sta proprio e "definitivamente» dentro la natura, immerso fisicamente e psichicamente in una attualità senza uscita. Certo, continua a cercare il motif, ma non più nel senso ottocentesco di tema sfizioso per una speciale emozione; invece come situazione essenziale ed oggettiva; così un contadinomontanaro cerca il luogo buono dove piantare o seminare qualcosa, un animale spia le tracce utili per seguire un percorso o marcare il territorio. Operaio, indossa perfino l'abito adatto e assume sulle spalle gli strumenti del lavoro, come aveva già fatto, ma ora esente da qualsiasi tentazione da dandy o da flâneur: così può sperare di compiere un lavoro ben fatto, attraverso un impegno che gli affatichi il corpo e gli occupi la mente per intero. E non c'è elegante parigina che si impanchi a spiegargli che la fatica affievolisce la concentrazione e inquina la sensibilità. È la condizione per così dire perfetta che ha desiderato e temuto per una vita (cultura e natura convergenti). Semmai resta il problema che ha assillato anche il grande modello: dove termini ciascun lavoro, addirittura se esista un termine per ciascun lavoro, visto che l'artificio si misura con la continuità delle naturali metamorfosi.

Ecco, allora, che diventa specialmente vivace l'incontro - quasi uno scontro - con la moglie. Per Nella, l'opera è importante, una "pietra miliare», lo è in quanto porta a compimento un'idea. L'occhio di Nella, allenato a cogliere il punto dove la visione tocchi necessaria epifania, vede anche nei lavori di Ugo il punto sufficiente, che può essere lontano dall'esito "perfetto", e cerca di convincerne il marito. Non è raro il caso che 
Nella sottragga ad Ugo uno stato che lui giudica approssimativo; come avviene che Ugo difenda le proprie ragioni vivacemente puntando risultati che la moglie non condivide perché li trova «troppo» e inutilmente complicati, o addirittura distrugga lavori espressivamente compiuti (del resto, lo si deduce dalle lettere a Bozzetti, il pittore non raramente ha vissuto l'esperienza di rovinare il «buono» già realizzato). Ma non dobbiamo sottovalutare l'aspetto etico del lavoro, che non può permettersi la scorciatoia, «il pressappoco che è peggio dell'errore», perché, come diceva l'amico fisiologo Beppino Levi (non dimentichiamo che Ugo ha una formazione scientifica) la scienza e anche l'arte - apparentate dall'essere forme di conoscenza - non valgono nulla per se stesse, ciò che nobilita l'uomo è il lavoro, la fatica e l'insoddisfazione dei risultati che eccita ulteriore ricerca.

Anche per Nella vale l'etica del lavoro, ma sono i risultati, per quanto provvisori, che contano, nel senso che, finalizzando l'impegno, lo giustificano e lo valorizzano. Il lavoro sta nel mezzo fra due assunzioni di responsabilità: la prima consiste nell'intuizione visionaria, la seconda nell'efficace proiettarsi della visione in figura essenziale. Sarebbe peccato disperdere la grazia della visione, sottraendola al suo destino di testimonianza. Detto in altro modo: l'artista ha una tremenda responsabilità, quella di travasare il vedere (sintetica intuizione in solitudine, visione che tocca esemplarmente Maria Annunciata) nel far vedere (manifestazione in oggetto della visione, la quale, dovendo «fissare» qualcosa di estremamente labile, non può che essere sintetica, nuda di qualsiasi distraente orpello).

Una giovane amica, poi pittrice, che posò spesso per Nella, testimonia che il vedere della Marchesini era piuttosto una proiezione che una constatazione. Dice Lia Rondelli che la pittrice era sostanzialmente indifferente al vedere immediato quanto inesorabile nel dichiarare la propria visione, non per imporla ma semplicemente perché ad essa non conosceva alternativa. Sempre Lia mi racconta di una visita con Nella alla Biennale di Venezia (esiste documento di una visita alla Biennale, dopo Padova e la Cappella degli Scrovegni nel '48). La Marchesini si porta appresso dovunque Le Confessioni di Sant'Agostino, legge sul treno, sul traghetto, passa senza accorgersene la fermata dei Giardini, scivola senza mostrare interesse particolare davanti a Picasso e Giacometti, forse ancora rimuginando la lettura che l'impegnava un attimo prima. Indifferenza, distrazione, depressione? Oppure concentrazione sul proprio mondo, esclusiva, dura, in un certo senso impietosa (di sé, non degli altri, che anzi verso gli altri, a cominciare dalla famiglia, la sua tolleranza era totale, senza eccezione). Non era possibile forzare quel mondo, rigorosamente sigillato non per egotismo — che anzi si stimava assai poco, e però riteneva doveroso 
non disperdere quel poco - né per egoismo — che anzi Nella non perseguiva interessi o vantaggi esclusivi - ma per senso del dovere, che solo meriterebbe onore e dignità ad una sopravvivenza altrimenti insensata. C'entrava, probabilmente, anche la sua religiosità, strettamente legata alla cognizione del dolore quanto alla speranza della redenzione. Redenzione non «meritata», concessa per grazia, però cercata desiderata dall'uomo poveretto. Letture privilegiate i libri morali della Bibbia, e poi i Padri della Chiesa, Jacopone da Todi, ecc.

Si dirà che quanto abbiamo finora detto c'entra assai poco con la pittura; sono convinto del contrario. L'unico modo di approssimarsi alla pittura di Nella è quello di aggirare, almeno in principio, i caratteri stilistici, i problemi formali, e di puntare decisamente sui contenuti umani e morali della sua pittura. Sono quei contenuti che le fanno accettare il rigoroso insegnamento di Casorati, perfino - non è un fatto minore la «liturgia» di accesso e operativa della pittura come viene presentata dal maestro. Il quale, infatti, non è tanto il detentore di una tecnica e di uno stile quanto un modello di moralità in arte, e di rigore, appunto liturgico, che presuppone una vera e propria iniziazione. Per questi motivi, tanto le appare remota e pur necessaria la figura del maestro/padre: sacerdote «triste e buono» che porta il peso di una iniziazione accettata che a sua volta tenta di travasare nel discepolo (non genericamente negli allievi ai quali può solo insegnare un mestiere). Necessario quel maestro, risorto dalla guerra e dai drammi famigliari, purificato dai birignao letterari di prima e dagli stilemi connessi, in una parola il Casorati gobettiano, quanto estraneo il modello formale del Casorati successivo, svuotato di quella «necessità dolorosa» che aveva improntato i primi anni di attività a Torino. E infatti sarà da quel ceppo che Nella svilupperà la sua pittura, fino a guadagnare una specie di espressionismo: da quello che sembra utilizzare Modigliani per raggiungere gli sguardi acuminati della pittura senese trecentesca, sul finire dei Venti inizio Trenta, fino alle ultime prove quasi segrete; ad un livello esemplare, a me pare altissimo: dagli autoritratti in figura di Vergine Annunciata alle «meditazioni» più che illustrazioni che accompagnano le Laude di Jacopone da Todi.

Gli anni Trenta e Quaranta vedono Nella e Ugo stretti da una condivisione piena dell'esistenza, ma anche della pittura, con tutti i distinguo del caso. È vero che la presenza pubblica della Marchesini si assottiglia, anche perché nel frattempo è venuta meno l'appartenenza alla «Scuola di Casorati» e le occasioni relative; ma non per questo cessa o si affievolisce l'impegno, sia pure soffertamente condizionato dalle incombenze famigliari. Semmai si può constatare che anche Nella rinuncia o almeno 
rimanda la realizzazione di quei capi d'opera che avevano caratterizzato almeno negli intenti il decennio precedente e che ricompariranno nel dopoguerra, peraltro nella maggioranza dei casi restando a livello di bozzettone aperto a molteplici possibilità. Ma anche qui ci si può chiedere se la scelta di materiali poveri, spesso di recupero, come cartone e compensato, non sia consapevole rifiuto di quella pienezza che le "opere» meriterebbero, ma che contraddirebbe il rigore dell'assunto etico; come è ragionevole chiedersi se non abbia avuto peso la vicinanza e l'esempio di Ugo, la sua scelta del "grigio» e di una aristocratica umiltà.

Per esempio, c'è un periodo ben preciso, che coincide con i lunghi soggiorni a Drusacco in Valchiusella specialmente durante la guerra ma anche dopo, nel quale la Marchesini dipinge quasi esclusivamente piccoli paesaggi e scene di fanciulli (figli e amici) bagnanti sul greto di un torrente. Veri e propri studi en plein air, non sembra in funzione di qualche opera a tema. Sono dunque, alla maniera di Nella mai integralmente naturalistica, motifs ripresi dal vero, perfino con qualche attenzione all'ora e alla stagione; ma sempre Nella "vede» più che guardare, tanto che si intuisce sottesa l'intenzione di ricondurre i dati ad una «storia». E questo, curiosamente, specie nei paesaggi, che assumono, pur nella loro modestia quantitativa, una valenza mitica: esemplare la serie dedicata al "Calvario», che non è denominazione topografica ma simbolica. Così anche le scene di registrazione famigliare spesso alludono a situazioni di contenuto "religioso».

Dalla parte di Ugo, certi temi quasi ossessivamente ripetuti in variante (un luogo del bosco, una svolta della strada, una casa, un fienile...) diventano a loro volta «mitici», come del resto era capitato al quasi modello Cézanne, sempre però tenendosi stretti alla osservazione analitica della natura. Non mancava un riferimento assai più vicino, e con il quale Ugo da lunga data e Nella da tempi più recenti intrattenevano rapporto profondo e continuativo, dico l'artista-filosofo Cino Bozzetti. Anche per Bozzetti, in piena coscienza e responsabilità, il problema era di far crescere la registrazione di un'immagine naturale fino ad una sintetica esemplarità, che solo lo schematismo verbale mette sotto insegna «simbolista». In realtà, la valenza simbolica non viene applicata artificiosamente, sta «nelle cose», la carica universale s'impone proprio nell'evidenza piena, "esagerata» delle cose. "Un primitivo è più universale di un parigino", arriva a dire l'ex parigino Malvano, mentre l'europeista Persico si chiede, proprio con riferimento a Bozzetti e Spazzapan, se la solitudine e la concentrazione mirata all'approfondimento non siano vie efficaci per la modernità, almeno quanto l'aggiornamento culturale. Nello stesso senso, più o meno, senza azzardare una «impossibile» conoscenza di quei testi da parte di 
Malvano, diventa illuminante la lettura di Cézanne comunicata da Rilke per via epistolare dopo la visita alla mostra del 1907. È un fatto che la lettura in chiave "realista» di Cézanne anziché secondo vettore "astratto» aiuta Malvano a non cadere nella trappola del formalismo. Diventano allora esemplari i disegni - solo disegni a matita su carta povera per mancanza di altri strumenti di lavoro - realizzati da Malvano a cominciare dall'estate del ' 44 alla primavera dell'anno successivo a Rosero sulla collina torinese, rifugio nel periodo conclusivo e particolarmente duro della guerra. In questi disegni, dedicati ai boschi cedui della zona tra Pecetto e Revigliasco, all'interno povero della camera dove la famiglia era ospitata e alle nature elementari (uova, patate, scarsi frutti, qualche recipiente) che il regime di stretta sopravvivenza concedeva, Malvano tocca esiti di impressionante verità, di magrezza materiale, che - mutatis mutandis - potrebbero confrontarsi con Morandi. E qui sovviene che tra le cose della prima maturità pittorica di Ugo, c'erano alcune nature morte dove il riferimento a Chardin era dichiarato, semmai rivisto attraverso più vicini riferimenti ai Nabis, in particolare Vuillard, cioè a modelli ch'erano valsi anche per Morandi (Morandi sfiorato dall'avanguardia, superata attraverso Cézanne e, a ritroso, Chardin e Corot).

Tra i pochi libri consultati a Rosero - ricorda ancora Lia Rondelli, la testimone cui abbiamo fatto riferimento diverse volte - c'era un libro di disegni di Cézanne. Una monografia su Cézanne (quella di Ambroise Vollard, Zurich, Crès, I919) che Ugo aveva regalato a Nella alcuni mesi prima del matrimonio, con la dedica «Alla consorella Nella Marchesini con molta amicizia e auguri. Dicembre I929». In quel momento, Cézanne, riconosciuto anche da Casorati come fondamentale incontro nel '2o (probabilmente più usato nei discorsi didattici che nella pittura), era l'unico punto di comune riferimento e reciproco riconoscimento. 